\title{
INDICADORES SUSTENTÁVEIS PARA O ESPAÇO URBANO: A REGIÃO METROPOLITANA DE SÃO PAULO
}

\author{
Claudio Noel de Toni Junior \\ Universidade Estadual Paulista - Unesp - Rio Claro, São Paulo, \\ Brasil \\ E-mail: junior_toni@terra.com.br
}

\begin{abstract}
Resumo: Esta pesquisa tem como objetivo a abordagem do conceito das cidades da Região Metropolitana de São Paulo (RMSP) através da criação do Índice de Sustentabilidade da Região Metropolitana de São Paulo (ISRMSP) por meio de dados de instituições como o Programa das Nações Unidas para o Desenvolvimento (PNUD), órgão vinculado a Organização das Nações Unidas (ONU) por meio do Atlas do Desenvolvimento Humano das cidades e do Índice de Desenvolvimento Humano Municipal (IDH-M) ao conjugar no Índice a ser criado, dois aspectos: o IDH-M e o Programa do Município da Cidade VerdeAzul (PMVA) através do Índice de Avaliação Ambiental (IAA) da Secretaria do Meio Ambiente do Estado de São Paulo que possui como atribuição desenvolver infraestrutura já existente nas cidades, por meio do avanço e aprimoramento do progresso técnico através da Ciência, da Tecnologia e da Informação ao modificar uma estrutura existente para otimizar processos produtivos em diversas variáveis como habitação, eletricidade, água potável para que haja bem-estar e qualidade de vida para a sociedade. A pesquisa incluiu as cidades da RMSP ao retratar o que pode ser melhorado em relação à educação em detrimento dos indicadores socioambientais. Por meio do uso de geotecnologias, como o programa ArcGis 10.1, serão elaborados mapas temáticos para estas cidades brasileiras, com o objetivo de abordar as múltiplas variáveis que demonstram a dinâmica da sustentabilidade e da qualidade de vida.
\end{abstract}

Palavras-chave: Urbanização. Sustentabilidade. Indicadores socioambientais. 


\section{RISUS - Journal on Innovation and Sustainability \\ ISSN 2179-3565}

RISUS - Journal on Innovation and Sustainability Volume 7, número $3-2016$

ISSN: 2179-3565

Editor Científico: Arnoldo José de Hoyos Guevara Editora Assistente: Nara Pamplona Macedo Avaliação: Melhores práticas editoriais da ANPAD

\title{
SUSTAINABILITY OF ENVIRONMENTAL INDICATORS FOR THE METROPOLITAN REGION OF SÃO PAULO
}

\author{
Kelly Carvalho Vieira, Valderí de Castro Alcântara, \\ José Willer do Prado, Paulo Henrique de Souza Bermejo \\ Universidade Federal de Lavras, Minas Gerais, Brasil \\ E-mail: kellycarvalho@posgrad.ufla.br, \\ valderi.alcantara@posgrad.ufla.br, jwprado@posgrad.ufla.br, \\ bermejo@dcc.ufla.br
}

\begin{abstract}
This research aims to bring the concept of cities in the metropolitan region of São Paulo ( MASP ) through the creation of the Sustainability Index of the Metropolitan Region of São Paulo ( ISRMSP ) through data from institutions like the United Nations Program for Development Program (UNDP), an agency of the United Nations ( UN) through the Atlas of Human Development of cities and the Municipal Human Development Index ( HDI -M ) combine the index to be created in two aspects : the HDI - M program and the City of Green City Blue ( PMVA) through the Environmental Assessment Index (WAI) from the Department of Environment of the State of São Paulo, which has as attribution develop existing infrastructure in cities, through the advancement and improvement of technical progress through Science, Technology and information to modify an existing structure to optimize production processes in various variables such as housing, electricity, drinking water so that there is well-being and quality of life for society. The survey included the cities of MRSP to portray what can be improved in relation to education at the expense of social and environmental indicators. Through the use of geotechnology, as the program Arcgis 10.1, thematic maps will be prepared for these Brazilian cities, with the objective of addressing the multiple variables that demonstrate the dynamics of sustainability and quality of life.
\end{abstract}

Keywords: Urbanization. environmental. sustainability indicators 


\section{INTRODUÇÃO}

A proposta inicial é apresentar os conceitos em uma abordagem socioambiental das cidades da Região Metropolitana de São Paulo (RMSP) por meio dos atributos socioambientais ao dinamizar o que já existe em termos de infraestrutura para melhorar e atingir patamares otimizados de melhoramento das atividades. Serão utilizadas tecnologias e as variáveis envolvidas, bem como a dinâmica da variabilidade ambiental que faz parte dos eventos ambientais tais como: desmoronamentos, mudanças climáticas, recursos naturais, áreas verdes degradadas, baixo nível de pegada ecológica, com biomas em situação desfavorável em termos da qualidade de vida no aspecto de vida multidimensional de um diagnóstico socioambiental.

Será apresentado o conceito de sustentabilidade em relação às cidades brasileiras mencionadas com o objetivo de realçar as disparidades socioambientais. O Índice de Sustentabilidade da Região Metropolitana de São Paulo (ISRMSP) propõe criar e divulgar para que haja melhorias que auxiliem os governos no aumento da qualidade de vida da população em estudo.

Neste sentido, espera-se que quanto maiores os benefícios de uma população em relação à qualidade de vida, maior será o índice de sustentabilidade das mesmas. Por consequência, a elaboração dos mapas temáticos indicará o nível de sustentabilidade em relação aos fatores mensuráveis: renda, nível de escolarização, saúde. $E$, também, os relacionados ao meio ambiente por meio do Programa do Município VerdeAzul (PMVA) através do Índice de Avaliação Ambiental (IAA) que analisa a vertente das cidades no que tange aos aspectos ambientais aliados à tecnologia e aos fatores socioeconômicos percebidos pelas pessoas residentes nestes municípios (GOVERNO, 2013 d).

Destaque os indicadores da RMSP do Programa das Nações Unidas (PNUD) órgão vinculado a Organização das Nações Unidas (ONU), mensura os aspectos socioeconômicos das cidades envolvidas dentre eles o índice de Desenvolvimento Humano Municipal (IDH-M)

\section{MATERIAIS E MÉTODOS}

A proposta que se faz nesta pesquisa é ir além do socioeconômico ao incluir o socioambiental, através do IAA, sendo que a junção do IDH-M das 39 cidades da RMSP trará a criação do Índice de Sustentabilidade da Região Metropolitana de São Paulo (ISRMSP). A padronização será através da unidade, quanto mais próximo de 1, 000 maior será o nível de sustentabilidade das cidades (PNUD, 2013b).

Para se alcançar o IAA, é utilizado o PMVA, programa voluntário que visa estimular os municípios ao desenvolvimento de estruturas no segmento ambiental por meio de dez diretivas: esgoto tratado, resíduos sólidos, biodiversidade, arborização urbana, educação ambiental, cidade sustentável, gestão das águas, qualidade do ar, estrutura ambiental e conselho ambiental (GOVERNO, 2013 d).

\section{RESULTADOS}

\section{Diálogo: Desenvolvimento e Sustentabilidade}

O conceito de sustentabilidade diz respeito a uma noção de aprendizagem colaborativa e interativa em que os agentes alicerçam suas experiências para colocá-las em prática, com o 
objetivo de obter ganhos positivos para toda a comunidade.

São organizadas através de projetos de governos e instituições para que seja mais sustentável à medida que usa ferramentas inovadoras em benefício da sociedade, além de aperfeiçoar a capacidade de infraestrutura já existente (GOVERNO, 2013a).

Mediante o uso inteligente de tecnologias socioambientais, como por exemplo, ruas que suportam cada vez mais automóveis, veículos limpos, para criar mecanismos de redução ligados a componentes que poluam menos e de projetos tecnológicos que planejem cidades através de uma conjuntura sustentável com condições para que as pessoas utilizem mecanismos alternativos, como as bicicletas, por exemplo.

Através da mobilidade e da integração, ressalta-se que as alternativas do progresso técnico estão inseridas no que denota sustentabilidade e ao Índice proposto e podem ser usadas de forma positiva para a redução dos poluentes como o $\mathrm{CO} 2$, alternativas qualitativas em termos de consumo de água, energia, transportes, aumento dos biomas, energia renovável.

Benson $(2008$, p. 21) trata da existência de sustentabilidade ao colocar a colaboração e a ênfase entre seus membros. Para a autora, tal conceito deve estar direcionado à construção e elaboração de competência múltiplas, cujo principal objetivo é a autorregulação, sendo primordial o desenvolvimento de metodologias e práticas que auxiliem a colaboração e a interação entre os envolvidos.

A educação é o primeiro passo, e o mais importante para que se tenham cidades integralizadas. Sendo assim, Benson (2008), Ferrada \& Flecha (2008) desenvolveram o conceito de Cidades de Aprendizagem com ênfase na educação, uma das variáveis do IDH-M. Como objetivos, tem-se: educação de qualidade, crianças matriculadas no respectivo ano escolar condizente com sua idade, professores capacitados e valorizados, jovens estimulados em aprender e o aprendizado valorizado e posto em prática na atualidade.

Sobretudo sirva para a construção de um pilar para toda a vida, mediante valores como: respeito às pessoas, dentre elas os idosos e a todas as classes. E não apenas prepará-los para uma carreira profissional; que esta seja além da base, mas também os pilares para que um profissional seja colaborativo, participativo e respeite a diversidade das pessoas em sua área de atuação e na vida como um todo (BENSON, 2008).

De acordo com Rosa, (2005):

"O Sistema de Processamento de Informações Georreferenciadas foi desenvolvido pela Divisão de Processamento de Imagens do Instituto Nacional de Pesquisas Espaciais (INPE). Trata-se de uma evolução dos sistemas SGI e SITIM, desenvolvidos para microcomputadores. Construído segundo o estado da arte em técnicas de modelagem e programação, combina uma interface com o usuário altamente interativa, interface de banco de dados que modela a metodologia de trabalho em estudos ambientais e manipulação unificada de dados espaciais, o que elimina o dilema raster-vector. Integra processamento de imagens, análise espacial e modelagem digital do terreno, além de interface com os bancos de dados. Opera em ambiente UNIX e Windows. É baseado num modelo de dados orientados-a-objetos, do qual são derivadas sua interface multi-janelas e a linguagem espacial". (p.88). 
As cidades da RMSP são cidades com forte crescimento econômico e social, principalmente no setor de serviços. Ao perder, no final de 1990, ganha importância no setor industrial com a migração de suas fábricas para cidades vizinhas, destaque para os segmentos de serviços como: comércio, pesquisa, alta tecnologia e a logística, todavia notam-se discrepâncias entre as mesmas no contexto social, econômico e socioambiental.

Nos dias atuais, predominam em sua economia os três setores: agricultura, indústria e serviços.

As cidades, objeto central em que se alicerça a pesquisa, possuem indicadores socioambientais positivos em detrimento de outras cidades do país como as inseridas na região Norte e Nordeste do Brasil.

A análise que se fará das cidades que permeiam a RMSP será baseada nas variáveis do IDH-M e no IAA que conjuga a interação com temas voltados aos indicadores e à variabilidade socioambiental, os quais realçarão os pilares para a construção do ISRMSP.

A aplicação do conceito de sustentabilidade é importante para que se possam fazer comparações, críticas e propor soluções para situações adversas de cidades com estrutura socioambiental dispersa. Nestas cidades, deve ser vista como uma perspectiva promissora para o crescimento e o desenvolvimento. E que a majoração destes indicadores os torne sustentáveis para que cada cidadão possa ter qualidade de vida.

Conforme Lombardo (1995):

"São as informações e dados sobre os problemas enfrentados pelos moradores, suas preferências, valores e suas qualidades atribuídas ao ambiente que embasarão as propostas de recuperação do meio ambiente urbano, que se fará através de um planejamento consciente, e consistente, tanto do seu aspecto físico como social".

A base da pesquisa consiste na análise dos indicadores socioambientais na esfera das cidades sustentáveis embasadas nas demais mencionadas, já que ao haver sustentabilidade, o ISRMSP, será cada vez mais positivo nos diversos cenários. Isto se dá porque a sustentabilidade é algo que aborda as nuances do conceito de qualidade de vida das pessoas e no que pode ser melhorada para atingir estes objetivos, como o Meio Ambiente, a Economia e a Geografia.

\section{Metodologia e Prática dos Indicadores Socioambientais para a construção do Índice de Sustentabilidade da Região Metropolitana de São Paulo}

Vale ressaltar que em termos do IDH-M, as cidades mencionadas figuram dispersas no ranking. Enquanto algumas ocupam as primeiras posições, outras estão em situação oposta e, quando se inclui o fator ambiental, as primeiras cidades no ranking serão aquelas, em que prevalecem políticas no que tange à qualidade de vida da região em análise. Em comparação aos valores observados no IDH-M das cidades base da pesquisa, observa-se que predominam na escala de alto IDH (de 0,700 a 0,799).

Porém, há cidades que possuem IDH muito alto, tais como as cidades de São Paulo, São Bernardo do Campo e São Caetano do Sul com IDH-M, respectivamente de: 0,805; 0,805 e 0,862. Quando analisadas sob a vertente do IAA, os destaques não estão nas cidades mencionadas, sendo Santo André a primeira no ranking na RMSP na 117ạ posição, São Paulo ocupa a 147ạ 
posição. As primeiras colocadas no ranking de 2011 foram: Santa Fé do Sul, Viradouro, Sorocaba, Fernandópolis e Guararapes (GOVERNO, 2013c).

O índice proposto a ser criado está representado por dados com função de disseminar a percepção das relações socioambientais por meio de indicadores disponibilizados por instituições variadas através de indicadores disponibilizados por diversos órgãos.

O ISRMSP oferece centenas de indicadores geofísicos da sociedade populacional, tais como: saúde, educação, renda, meio ambiente ao mostrar, através de uma proxy que se emprega o Índice sintético calculado matematicamente mediante informações coletadas. Por exemplo, em termos da variável saúde, são utilizados dados da longevidade da população, sendo esta a proxy que se emprega no valor da variável em questão (PNUD, 2010b).

A criação do ISRMSP é primordial para que seja ampliada a concepção de desenvolvimento sustentável. Acredita-se que o IDH-M seja um Índice satisfatório, porém mensura de forma parcial o desenvolvimento humano. Acrescentar indicadores socioambientais é importante para que o desenvolvimento seja alicerçado em novas variáveis que possam retratar com maior realidade o desenvolvimento percebido pela sociedade. Por isso, na criação do ISRMSP serão adotados índices socioambientais que geram qualidade de vida (TONI JUNIOR, 2013).

Por trás da variável saúde, existe uma gama de outros indicadores, pois nota-se que uma população com acesso a serviços básicos essenciais, ligados à qualidade de vida, terá uma esperança de vida maior. Dentre tais indicadores podemos citar: acesso à saúde de qualidade nos hospitais, erradicação da mortalidade infantil e pré-natal, moradias adequadas com acesso a saneamento básico, água potável, trabalho em condições saudáveis, não insalubres, acesso a cultura, lazer, esporte, fatores que trarão bem-estar econômico e social.

Em outras palavras, uma sociedade sustentável, trará os benefícios do progresso técnico e científico, as pessoas terão maior grau de escolarização, com isso, tendencialmente terão empregos com renda que lhes garantam a sustentabilidade econômica, que por consequência, terão acesso a bens sociais majorados, como, por exemplo, moradia digna, plano de saúde, ou seja, acesso a condições de saúde satisfatórias (PNUD, 2011b).

O Atlas com seus dados propõem uma análise diversificada das dimensões, mediante os rankings existentes. (PNUD, 2010a). Pode-se citar um exemplo, as cidades ricas, em termos de população, que eram duas em 1960, a saber: São Paulo e Rio de Janeiro, tornam-se cinco em 1970, dez em 1980 e doze em 1991.

Neste novo contexto de urbanização ou da metropolização ou quase conurbação que se aproxima dessa margem, tal número será majorado (Lombardo, 1995 apud Santos, 1993). A criação do ISRMSP possui o objetivo de ser um Índice que forneça bases conceituais para que se promovam as bases de análise e de propostas inovadoras de melhorias contínuas ao verificar o que se pode melhorar em termos da estrutura socioambiental da RMSP.

Devido à inquietação cada vez mais eminente com a temática sobre questões ambientais, como poluição, desmatamento e áreas verdes, dentre outras, além das consequências de efeitos negativos como destruição de florestas, perda de mananciais, enfim, os malefícios causados na biodiversidade mundial, criaram-se doutrinas para que haja debates acadêmicos e cúpulas internacionais.

Menciona os diversos e variados encontros que abordaram a questão do meio ambiente 
como as Conferências Mundiais, tais como a Rio +20 em que estiveram em voga temas e teorias para que a questão da sustentabilidade fosse abordada através de um enfoque de perspectivas de melhorias ao longo dos anos mediante metas para que a humanidade não careça de perdas consideráveis de seu habitat (PNUD, 2011a apud TONI JUNIOR, 2013)

No relatório de Desenvolvimento Humano (RDH) publicado pelo Programa das Nações Unidas (PNUD) no Brasil, órgão vinculado a ONU nos anos de 2007 e 2008, constatou-se a necessidade de analisar, mensurar e propor alternativas para o que se entenda de valores humanos.

Uma campanha foi realizada através de uma pergunta, O que precisa mudar no Brasil para a sua vida melhorar de verdade? Esta pergunta chegou aos brasileiros por meio de etapas por meio de perguntas diretas, inicialmente nos municípios que possuem os piores índices de IDH e posteriormente através da média por meios de comunicação com: rádio, TV, internet, celular, etc. Estima-se que aproximadamente 500 mil pessoas responderam esta pergunta. (BRASIL, PNUD, 2007/08).

Esta pergunta teve a intenção de dimensionar o que os brasileiros pensam em relação aos problemas sociais por eles enfrentados como a falta de educação básica e a ausência de oportunidades de frequentar uma universidade, por exemplo.

A ineficiência ou até mesmo a exclusão de serviços de saneamento básico como a água potável e esgoto, o impacto negativo em relação à perda da biodiversidade ocasionada por meio da poluição, aquática, do ar e dos solos, relação à perda da qualidade de vida das pessoas.

A RMSP pode ser considerada pode ser considerado o maior conglomerado de riqueza do país, ou seja, de renda, mensurada pelo PIB pelos municípios que a compõe, ultrapassando mais de $50 \%$ a riqueza do estado de São Paulo, conforme a tabela

TABELA 1 - IDH-M (2010) E SUAS VARIÁVEIS

\begin{tabular}{c|c|c|c|c} 
LUGAR & IDHM 2010 & IDHM Renda 2010 & $\begin{array}{c}\text { IDHM Longevidade } \\
2010\end{array}$ & $\begin{array}{c}\text { IDHM- Educa- } \\
\text { ção 2010 }\end{array}$ \\
\hline Arujá (SP) & 0,727 & 0,739 & 0,816 & 0,637 \\
\hline Barueri (SP) & 0,784 & 0,761 & 0,866 & 0,73 \\
\hline Biritiba-Mirim0,712 & 0,786 & 0,791 & 0,866 & 0,708 \\
\hline Caieiras (SP) & 0,712 & 0,71 & 0,795 & 0,64 \\
\hline Caieiras (SP) & 0,781 & 0,74 & 0,861 & 0,749 \\
\hline Cajamar (SP) & 0,728 & 0,713 & 0,81 & 0,668 \\
\hline Carapicuíba (SP) & 0,749 & 0,721 & 0,842 & 0,693 \\
\hline Cotia (SP) & 0,78 & 0,789 & 0,851 & 0,707 \\
\hline Diadema (SP) & 0,757 & 0,717 & 0,844 & 0,716 \\
\hline Embu (SP) & 0,735 & 0,7 & 0,839 & 0,676 \\
\hline Embu-Guaçu (SP) & 0,749 & 0,713 & 0,834 & 0,708 \\
\hline Ferraz de Vasconcelos & 0,738 & 0,691 & 0,828 & 0,703 \\
\hline (SP) & & 0,659 & 0,815 & 0,647 \\
\hline Francisco Morato (SP) & 0,703 & 0,702 & 0,852 & 0,654 \\
\hline Franco da Rocha (SP) & 0,731 & & &
\end{tabular}




\begin{tabular}{|c|c|c|c|c|}
\hline Guararema (SP) & 0,731 & 0,729 & 0,817 & 0,656 \\
\hline Guarulhos (SP) & 0,763 & 0,746 & 0,831 & 0,717 \\
\hline Itapevi (SP) & 0,735 & 0,687 & 0,855 & 0,677 \\
\hline Itapecerica da Serra (SP) & 0,742 & 0,699 & 0,852 & 0,687 \\
\hline Itaquaquecetuba (SP) & 0,714 & 0,665 & 0,844 & 0,648 \\
\hline Jandira (SP) & 0,76 & 0,738 & 0,841 & 0,706 \\
\hline Juquitiba (SP) & 0,709 & 0,68 & 0,791 & 0,662 \\
\hline Mairiporã (SP) & 0,788 & 0,767 & 0,881 & 0,723 \\
\hline Mauá (SP) & 0,766 & 0,721 & 0,852 & 0,733 \\
\hline Mogi das Cruzes (SP) & 0,783 & 0,762 & 0,851 & 0,74 \\
\hline Osasco (SP) & 0,776 & 0,776 & 0,84 & 0,718 \\
\hline $\begin{array}{c}\text { Pirapora do Bom Jesus } \\
\text { (SP) }\end{array}$ & 0,727 & 0,679 & 0,81 & 0,698 \\
\hline Poá (SP) & 0,771 & 0,71 & 0,856 & 0,754 \\
\hline Ribeirão Pires (SP) & 0,784 & 0,749 & 0,847 & 0,76 \\
\hline Rio Grande da Serra (SP) & 0,749 & 0,684 & 0,823 & 0,745 \\
\hline Salesópolis (SP) & 0,732 & 0,687 & 0,829 & 0,69 \\
\hline Santa Isabel (SP) & 0,738 & 0,7 & 0,834 & 0,689 \\
\hline $\begin{array}{c}\text { Santana de Parnaíba } \\
\text { (SP) }\end{array}$ & 0,814 & 0,876 & 0,849 & 0,725 \\
\hline Santo André (SP) & 0,815 & 0,819 & 0,861 & 0,769 \\
\hline $\begin{array}{c}\text { São Bernardo do Cam- } \\
\text { po (SP) }\end{array}$ & 0,805 & 0,807 & 0,861 & 0,752 \\
\hline São Caetano do Sul (SP) & 0,862 & 0,891 & 0,887 & 0,811 \\
\hline $\begin{array}{c}\text { São Lourenço da Serra } \\
\text { (SP) }\end{array}$ & 0,728 & 0,704 & 0,823 & 0,666 \\
\hline São Paulo (SP) & 0,805 & 0,843 & 0,855 & 0,725 \\
\hline Suzano (SP) & 0,765 & 0,708 & 0,873 & 0,723 \\
\hline Taboão da Serra (SP) & 0,769 & 0,742 & 0,863 & 0,71 \\
\hline $\begin{array}{c}\text { Vargem Grande Paulista } \\
\text { (SP) }\end{array}$ & 0,77 & 0,755 & 0,884 & 0,683 \\
\hline
\end{tabular}

Fonte: Atlas do Desenvolvimento Humano. Programa das Nações Unidas para o Desenvolvimento, PNUD. (2010).

Pelos dados do Atlas percebe-se as estatísticas do IDH do ano de 2010 divulgado no Atlas de Desenvolvimento Humano de 2013, onde situa-se os dados de cada município da RMSP, percebe-se que mesmo pertencendo a mesma região metropolitana, há municípios com valores superiores aos demais, tais como, os municípios do ABC em comparação com cidades como: Ferraz de Vasconcelos, Francisco Morato, Itaquaquecetuba, entre outros com valores inferiores.

Desta maneira, veem-se municípios com melhor classificação, sendo que muitos não possuem o PIB de grandes cidades como cidade de São Paulo, por exemplo, país possui variáveis sócias positivas como a saúde e a educação, por isto encontra-se em uma posição superior no ranking do IDH, conforme se pode visualizar no mapa 1, onde mediante o software ArcGis 10.1 
como um realce do uso da geotecnologia para que o leitor tenha a perspectiva geográfica do espaço abordado.

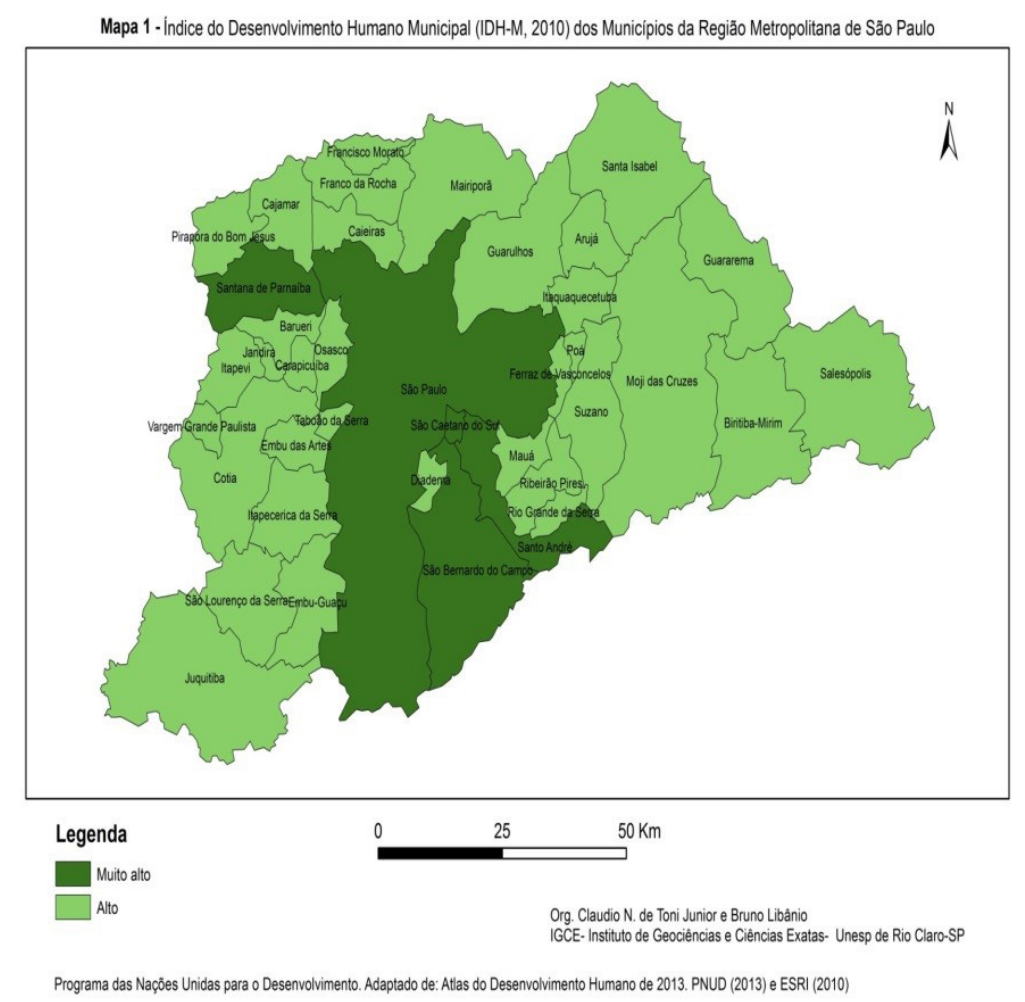

Mediante o uso do SIG, criou-se o mapa onde se observa que as cidades com o IDH muito alto estão dispersas em relação aos municípios com IDH alto.

Percebe-se que por pertencer a uma das regiões com maior nível de estrutura socioeconômica do país não se observou cidades com IDH médio ou baixo. Destaque para as cidades de: São Paulo, Santana do Parnaíba, São Bernardo do Campo e São Caetano do Sul que se sobressaíram sobre os demais municípios.

Com relação à noção do espaço e do tempo, Martin e Frugol Junior (1992) na obra é "Braz do Brazil, Brás de todo o mundo" (Pagu) (*) menciona e disserta o espaço da capital paulista em um local específico demonstrando suas particularidades, aspectos da cultura, da vivência da sociedade da época e sua evolução ao longo dos anos com a introdução de mecanismo inovadores que mudaram um estilo de vida enraizado, cita as ferrovias do Brás, os aspectos da sociedade de outros bairros paulistanos, como o da Freguesias do Ó mediante uma linha histórica temporal onde de forma gradual foi se estabelecendo pomares de cultura frutíferas, sítios, hortas, menciona o abastecimento do comércio na rua das "Casinhas" 1 no centro ou as chácaras residenciais em seu entorno neste período de tempo que prepara século XIX até o século XX.

A análise que se fará nas cidades que permeiam a RMSP será baseada nas variáveis do IDH-M e no PMVA que conjuga a interação com temas voltados aos indicadores socioambientais, que irá realçar os pilares para a construção do ISRMSP. 
A aplicação do conceito de sustentabilidade é importante para que se possa fazer comparações, críticas e propor soluções para situação adversas de cidades com estrutura socioambiental dispersas. Nestas cidades deve ser vista como uma perspectiva promissora para o crescimento e o desenvolvimento das mesmas. E que a majoração destes indicadores as torne sustentável para que cada membro da mesma tenha qualidade de vida.

Na observação da tabela 2, verifica-se os dados obtidos no ranking do Índice final do programa do PMVA, dos municípios da RMSP para o ano de 2011, padronizados pela porcentagem.

TABELA 2 - ÍNDICE de AVALIAÇÃO AMBIENTAL do PROGRAMA dO MUNICÍPIO VERDEAZUL DA REGIÃO METROPOLITANA DE SÃO PAULO (2011)

\begin{tabular}{|c|c|}
\hline Arujá (SP) & 0,5762 \\
\hline Barueri (SP) & 0,6056 \\
\hline Biritiba-Mirim (SP) & 0,1640 \\
\hline Caieiras (SP) & 0,3988 \\
\hline Cajamar (SP) & 0,0574 \\
\hline Carapicuíba (SP) & 0,0110 \\
\hline Cotia (SP) & 0,4417 \\
\hline Diadema (SP) & 0,4391 \\
\hline Embu (SP) & 0,6587 \\
\hline Embu-Guaçu (SP) & 0,0871 \\
\hline Ferraz de Vasconcelos (SP) & 0,0828 \\
\hline Francisco Morato (SP) & 0,0541 \\
\hline Franco da Rocha (SP) & 0,786 \\
\hline Guararema (SP) & 0,6955 \\
\hline Guarulhos (SP) & 0,5830 \\
\hline Itapevi (SP) & 0,01768 \\
\hline Itapecerica da Serra (SP) & 0,1752 \\
\hline Itaquaquecetuba (SP) & 0,0163 \\
\hline Jandira (SP) & 0,0156 \\
\hline Juquitiba (SP) & 0,1198 \\
\hline Mairiporã (SP) & 0,5112 \\
\hline Mauá (SP) & 0,4088 \\
\hline Mogi das Cruzes (SP) & 0,4482 \\
\hline Osasco (SP) & 0,4436 \\
\hline Pirapora do Bom Jesus (SP) & 0,0784 \\
\hline Poá (SP) & 0,1743 \\
\hline Ribeirão Pires (SP) & 0,8117 \\
\hline Rio Grande da Serra (SP) & 0,2786 \\
\hline Salesópolis (SP) & 0,8557 \\
\hline Santa Isabel (SP) & 0,3750 \\
\hline Santana de Parnaíba (SP) & 0,4925 \\
\hline
\end{tabular}




\begin{tabular}{c|c}
\hline Santo André (SP) & 0,8220 \\
\hline São Bernardo do Campo (SP) & 0,5946 \\
\hline São Caetano do Sul (SP) & 0,8479 \\
\hline São Lourenço da Serra (SP) & 0,1294 \\
\hline São Paulo (SP) & 0,8037 \\
\hline Suzano (SP) & 0,5357 \\
\hline Taboão da Serra (SP) & 0,6188 \\
\hline Vargem Grande Paulista (SP) & 0,0703
\end{tabular}

Fonte: Programa do Município VerdeAzul da Secretaria do Meio Ambiente (SMA). São Paulo. 2011

Conforme dos dados da tabela 2, realça a média obtida em todas as diretivas ao ser alcançado o Índice geral do PMVA com ênfase ao IAA para o ano de 2011.

O PMVA da Secretaria do Meio Ambiente do Estado de São Paulo do meio ambiente (SMA) tem a intenção mediante parâmetros similares que envolvem os 654 município do estado de São Paulo de que o poder das instituições como os governos seja motivado a prover alternativas de melhorias no que tange ao ambiente de cada município.

Destaca que as diferenças em suas estruturas no segmento orçamentário que há entre os municípios faz com que se tenham planos de natureza ambiental com estruturas diferentes. Porem existe normalização mínima para a análise de padrões estatísticos mínimos dos indicadores envolvidos.

Desta maneira, os municípios que participam deste programa passam a ter uma dinâmica que avaliam os quesitos inerentes ao meio ambiente de uma forma mais ampla e faz com que os órgãos estaduais competentes que trabalham para a melhoria das variáveis sustentáveis do meio ambiente criem condições satisfatórias para questões ambientais e que seja parte integrante das estruturas que envolvem as relações em sociedade no espaço e no tempo.

A base da pesquisa é analisar os indicadores socioambientais na esfera das cidades sustentáveis embasadas nas cidades citadas, já que ao haver sustentabilidade, o ISRMSP, será cada vez mais positivo nos diversos cenários. Isto se dá porque a sustentabilidade é algo que aborda as nuances do conceito de qualidade de vida das pessoas e no que pode ser melhorada para atingir estes objetivos, como o Meio Ambiente, a Economia e a Sociologia.

Vale ressaltar que, em termos do IDH-M, as cidades mencionadas, figuram dispersas no ranking. Enquanto algumas estão nas primeiras posições, outras estão em situação oposta e quando se inclui o fator ambiental as primeiras cidades no ranking serão aquelas, que prevalecem políticas no que tange a qualidade de vida da região em análise.

Representado por dados com função de disseminar a percepção das relações socioambiental por meio de indicadores disponibilizados por instituições variadas através de indicadores disponibilizados por órgãos nacionais e internacionais.

A técnica é um meio de interação do homem com o meio natural. Analisa e fundamenta uma questão epistemológica e propõe alternativas críticas ao dar uma resposta à questão do desenvolvimento (IANNI, et al., 1995, apud TONI JUNIOR, 2013).

Mediante a abordagem do espaço e sua relação com o tempo, aliar-se-ão os conceitos socioambientais, com base teórica no espaço e do geoprocessamento de dados através da elaboração de mapas temáticos. 
A questão da sustentabilidade é um dos maiores objetivos e desafios da pesquisa, sendo a criação desta temática no âmbito das cidades mencionadas. Para isto será usada à temática metodológica de cientistas que estudam a sustentabilidade em nível local e regional, principalmente, para ser um dos pilares da pesquisa para a concepção do ISRMSP para as cidades.

A criação do ISRMSP foi baseada na soma do Índice do PMVA 2011 e do IDH-M 2010, de acordo com a metodologia IDH-M+ IAA/2 com o objetivo de ser criado um Índice que contempla um indicador socioambiental sendo o conjunto das variáveis econômicas, sociais e ambientais, conforme tabela 3.

TABELA 3 - ÍNDICE DE SUSTENTABILIDADE DA REGIÃO METROPOLITANA DE SÃO PAULO (ISRMSP - 2013).

\begin{tabular}{|c|c|}
\hline Arujá (SP) & 0,6801 \\
\hline Barueri (SP) & 0,695 \\
\hline Biritiba-Mirim (SP) & 0,438 \\
\hline Caieiras (SP) & 0,589 \\
\hline Cajamar (SP) & 0,392 \\
\hline Carapicuíba (SP) & 0,380 \\
\hline Cotia (SP) & 0,610 \\
\hline Diadema (SP) & 0,598 \\
\hline Embu (SP) & 0,696 \\
\hline Embu-Guaçu (SP) & 0,418 \\
\hline Ferraz de Vasconcelos (SP) & 0,410 \\
\hline Francisco Morato (SP) & 0,378 \\
\hline Franco da Rocha (SP) & 0,404 \\
\hline Guararema (SP) & 0,713 \\
\hline Guarulhos (SP) & 0,673 \\
\hline Itapevi (SP) & 0,376 \\
\hline Itapecerica da Serra (SP) & 0,458 \\
\hline Itaquaquecetuba (SP) & 0,365 \\
\hline Jandira (SP) & 0,387 \\
\hline Juquitiba (SP) & 0,414 \\
\hline Mairiporã (SP) & 0,649 \\
\hline Mauá (SP) & 0,587 \\
\hline Mogi das Cruzes (SP) & 0,615 \\
\hline Osasco (SP) & 0,609 \\
\hline Pirapora do Bom Jesus (SP) & 0,402 \\
\hline Poá (SP) & 0,472 \\
\hline Ribeirão Pires (SP) & 0,797 \\
\hline Rio Grande da Serra (SP) & 0,513 \\
\hline Salesópolis (SP) & 0,793 \\
\hline Santa Isabel (SP) & 0,556 \\
\hline Santana de Parnaíba (SP) & 0,653 \\
\hline
\end{tabular}




\begin{tabular}{c|c}
\hline Santo André (SP) & 0,818 \\
\hline São Bernardo do Campo (SP) & 0,699 \\
\hline São Caetano do Sul (SP) & 0,854 \\
\hline São Lourenço da Serra (SP) & 0,428 \\
\hline São Paulo (SP) & 0,804 \\
\hline Suzano (SP) & 0,650 \\
\hline Taboão da Serra (SP) & 0,693 \\
\hline Vargem Grande Paulista (SP) & 0,420
\end{tabular}

Fonte: Programa do Município VerdeAzul da Secretaria do Meio Ambiente do Estado de São Paulo (SMA, 2011).

A proposta é analisar e propor alternativas de índices ligados a cada um destes municípios, ao abranger aspectos intrínsecos da sustentabilidade e de variáveis como: renda, saúde, educação, habitação, água potável, energia, meio ambiente, e os avanços do progresso técnico através da Ciência, da Tecnologia e da Informação no que se refere a melhorias na qualidade de vida das pessoas em que a pesquisa se alicerça principalmente no que se refere a variável educação que será comparada em relação ao ISRMSP por meio de vertentes como as Cidades Inteligentes (CI) e Cidades da Aprendizagem.

Estes fatores estão ligados à sustentabilidade, e além do IDH-M, a análise de estudo está embasada na problemática de índices ligados ao meio ambiente, comparando-os com os índices socioeconômicos do IDH-M.

A consulta na plataforma é realizada através de dados estatísticos disponíveis, por meio de mapas de cada um dos municípios abordados, seu perfil que demonstra uma realidade vivenciada nos últimos 20 anos do IDHM e dos 180 indicadores disponíveis.

O ranking foi elaborado para possibilitado para possibilitar a consulta do Índice onde se pode fazer comparações, discussões e verificar qual das variáveis que precisa de melhorias quando se compara um município ao outro.

Em colaboração com a Masschussets Intitute Of Technology (MIT), a Universidade de Harvard e a Northianstern Universty houve a elaboração visual do IDHM no Brasil a partir de dados quantitativos em forma de gráficos, mapas em formato de árvore, onde a altura do tronco da árvore pode representar o Índice e as variáveis do IDHM os "galhos" que representam o tamanho dos blocos ao proporcionar os valores da renda, saúde e educação.

Desta maneira percebe-se que houve a participação de Institutos e Universidade internacionais, que colaboraram para que houvesse a criação de forma qualitativa do Atlas, ou seja, o auxílio foi fundamental para que as instituições brasileiras pudessem desenvolver esta plataforma.

\section{CONSIDERAÇÕES FINAIS}

A formulação da pesquisa foi realizada por meio de leituras de obras que abordam inicialmente o espaço geográfico e a inserção do homem no contexto da globalização e sua relação com a tecnologia através de autores que versam sobre o desenvolvimento e sua interação com os indicadores socioambientais.

Será realizada a definição do IDH-M, a questão do meio ambiente como a poluição que abordam a sustentabilidade aliados a educação e a qualidade de vida, o uso das geotecnologias 
aplicadas será um facilitador para que se perceba o espaço com melhor amplitude e riqueza de detalhes.

Os temas abordados anteriormente estão intrinsecamente ligados ao tema da Informação e da Inovação. Ciência está ligada à educação, cultura, enfim variáveis sustentáveis por meio de instituições tais como a ONU mediante a divulgação do IDH-M pelo PNUD e pelos dados da Secretaria do Meio Ambiente do Estado de São Paulo.

Educação de qualidade fará com que o Brasil avance na Ciência e na Tecnologia em múltiplos fatores, como na tecnologia digital, eletrônica, mecatrônica, dentre outras.

Da mesma forma que políticas de sustentabilidade, as quais tenham por objetivo a preservação ambiental, ter-se-á uma sociedade que evolua e tenha bem-estar socioeconômico e ambiental (PUNUD, 2011b). 


\section{REFERÊNCIAS BIBLIOGRÁFICAS}

BENSON, B. (2008). How to Motivate Students, Meet Standards, and Still Enjoy Teaching: Four Practices That Improve Student Learning. Disponível em:

http://books.google.pt/books?id=TmMFLmVdWEgC\&dq=Barbara+Benson,+How+to+Moti vate+Students,+Meet+Standards,+and+Still+Enjoy+Teaching\&printsec=frontcover\&source =bl\&ots=Xz4EnNoOUd\&sig=C1aeGqtQsZwHR4T-mD5qtzWubsM\&hl=pt-PT\&ei=ZvK3SrjYNI_ SjAeA7azZCw\&sa=X\&oi=book. Acesso em: 15 mar. 2015

BRASIL, Instituto Brasileiro de Geografia e Estatística (IBGE). Censo Demográfico 2010. Disponível em: http://www.censo2010.ibge.gov.br/apps/mapa/. Acesso em: 12 mai. 2013.

GOVERNO, Secretaria do Meio Ambiente do estado de São Paulo. Município VerdeAzul. Disponível em: < http://www.ambiente.sp.gov.br/municipioverdeazul/files/2013/03/Manual_PMVA_2013. pdf>.Acesso em : 02set .2015.

LOMBARDO, M.A. QUALIDADE AMBIENTAL E PLANEJAMENTO URBANO: Considerações de Método. Tese de Livre Docência. Faculdade de Filosofia, Letras e Ciências Humanas - FFLCH da Universidade de São Paulo, 267 p. São Paulo. 1995.

PROGRAMA DAS NAÇÕES UNIDAS PARA O DESENVOLVIMENTO (PNUD). Relatório do desenvolvimento humano. 2010a. Disponível em: http://www.pnud.org.br/raca/reportagens/ index.php?id01=3437\&lay=rac

Acessado em: 22 mai.2015.

.Relatório do Desenvolvimento Humano. 2010b A Verdadeira Riqueza das Nações: Vias para o Desenvolvimento Humano. Disponível em: <http://hdr.undp.org/en/reports/global/ hdr2010/chapters/pt/

Acessado em: 25 mai.2013>.

.Relatório do desenvolvimento humano. 2011a. Disponível em:

< http://hdr.undp.org/en/media/HDR_2011_PT_Complete.pdf>. Acesso em: 28 mar. 2015.

Responder aos desafios políticos. 2011b. Disponível em: <http://hdr.undp.org/ en/media/HDR_2011_PT_Chapter5.pdf>. Acesso em: 27. set.2012.

.Atlas do Desenvolvimento Humano no Brasil.2013 . Disponível em: <http://www. pnud.org.br/IDH/Atlas2013.aspx?indiceAccordion=1\&li=li_Atlas2013>. Acesso em: 14 set.2013.

ROSA, R. Geotechnologieson applied geographie. Revista do Departamento de Geografia, n. 16, p. 81-90. 2005.

SANTOS, M. A natureza do espaço: técnica e tempo, razão e emoção. 4. ed. São Paulo: Edusp, 2006. 
SÃO PAULO, Governo do Estado de São Paulo. Secretaria estadual do Meio Ambiente. Município VerdeAzul. Disponível em: <http://www.ambiente.sp.gov.br/municipioverdeazul/rankingpontuacao/>. Acesso em: 12 ago.2013.

TONI JUNIOR, C. N. Análise de indicadores metodológicos de sustentabilidade socioambiental. 273 f. 2013. Tese (Doutorado em Geografia). - Universidade Estadual Paulista “Júlio de Mesquita Filho", Rio Claro, 2013. 